Journal of Applied Fluid Mechanics, Vol. 14, No. 5, pp. 1535-1546, 2021. Available online at www.jafmonline.net, ISSN 1735-3572, EISSN 1735-3645. https://doi.org/10.47176/jafm.14.05.32251

\title{
Flow and Heat Transfer Performance of Channels with 45 Degree Ribs in Staggered Array
}

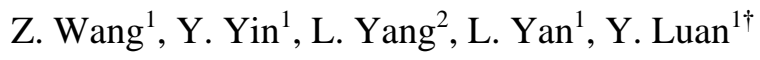 \\ ${ }^{1}$ College of Power and Energy Engineering, Harbin Engineering University, Harbin, Heilongjiang Province, \\ 150001, China \\ ${ }^{2}$ DIME - Department of Mechanical, Energy, Management and Transportation Engineering, University of \\ Genoa, Via Montallegro 1, 16145 Genova, Italy
}

†Corresponding Author Email: Shandong-313@163.com

(Received September 28, 2020; accepted February 2, 2021)

\begin{abstract}
The cooling efficiency of blade is growing demand with increasing turbine inlet temperature in gas turbine development. Ribs used in cooling channels is a common cooling structure, therefore, many configurations were studied by previous literatures, including angle, spacing, shape etc. However, there are less research about the dislocation ribs structure. In this paper, the 45-deg parallel ribs, crossed ribs and dislocation ribs were investigated by numerical simulation, in order to reveal the heat transfer performance and flow mechanism. Refer to the experiment, SST k- $\omega$ model was applied in steady simulation, at Re from 20000 to 50000. Due to the angled ribs can induce the secondary flow and generate small helical vortices at front corner, heat transfer performance was elevated. The large rotating vortices influenced by the ribs arrangement occupy the center channel, thence the dislocation caused different flow and heat transfer results. The results shown that parallel rib has higher heat transfer enhancement than crossed ribs, but pressure loss possess considerable level. At $\mathrm{Re}=21587$, the averaged turbulent kinetic energy of Case 2.2 with dislocation ribs is $22.4 \%$ lower than parallel ribs. The all 45-deg crossed ribs present higher level of overall thermal performance, and Case 2.2 is optimal for the range of Re investigated.
\end{abstract}

Keywords: Internal cooling; Numerical simulation; Ribs; Gas turbine; Heat transfer.

\section{NOMENCLATURE}

$\begin{array}{ll}C_{\mathrm{p}} & \text { specific heat at constant pressure } \\ D_{h} & \text { inlet hydraulic diameter } \\ d & \text { thickness of plate } \\ e & \text { Height of ribs } \\ f & \text { friction factor } \\ f_{0} & \text { friction factor in smooth channel } \\ H & \text { height of pin fin } \\ h & \text { heat transfer coefficient } \\ k & \text { turbulence kinetic energy } \\ k l & \text { kinetic energy of laminar } \\ L & \text { streamwise length of the channel } \\ N u & \text { Nusselt number } \\ N u_{0} & \text { Nusselt number in smooth channel } \\ R e & \text { Reynolds number } \\ \mathrm{RNG} & \text { Re- normalization group } \\ \mathrm{SST} & \text { Shear Stress Transfer } \\ T & \text { the temperature }\end{array}$

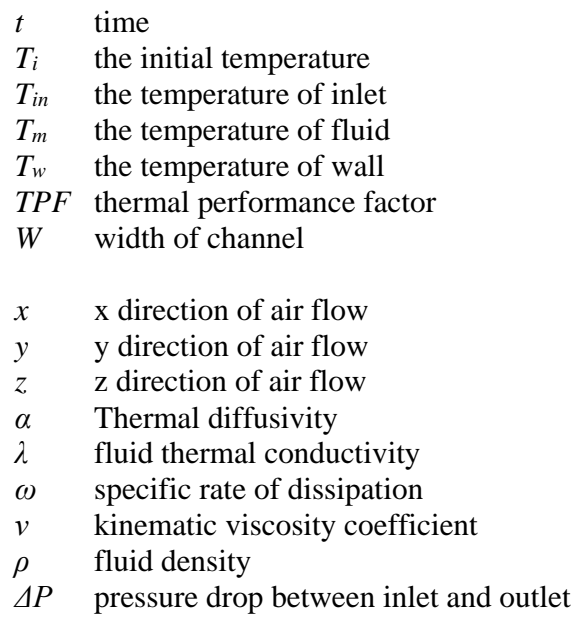




\section{INTRODUCTION}

Gas turbine is a kind of high-energy density engine and improving thermodynamic efficiency has been a major topic in industry for decades. Increasing turbine inlet temperature and pressure is the most direct way to achieve the goal, however, it puts forward higher requirements for blade heat resistance and strength. Compared with developing high-performance turbine blade matrix materials, the research period of designing and adopting advanced cooling measures is shorter and the investment is less. For example, the impingement cooling, convective cooling and pin-fin cooling are capable of maintaining the blade temperature at a relatively low level through increasing the heat transfer rate between internal walls and coolant. In mass of studies, a well-known method to increase heat transfer is to employ the rib turbulators in the chord region of blade. As shown in Fig. 1(a), the ribbed structure occupies most of core area inside the practical blade (Han and Rallabandi 2010), and Fig. 1(b) shows the mostly mentioned rib types in papers: parallel, angled, criss-cross and chevron.

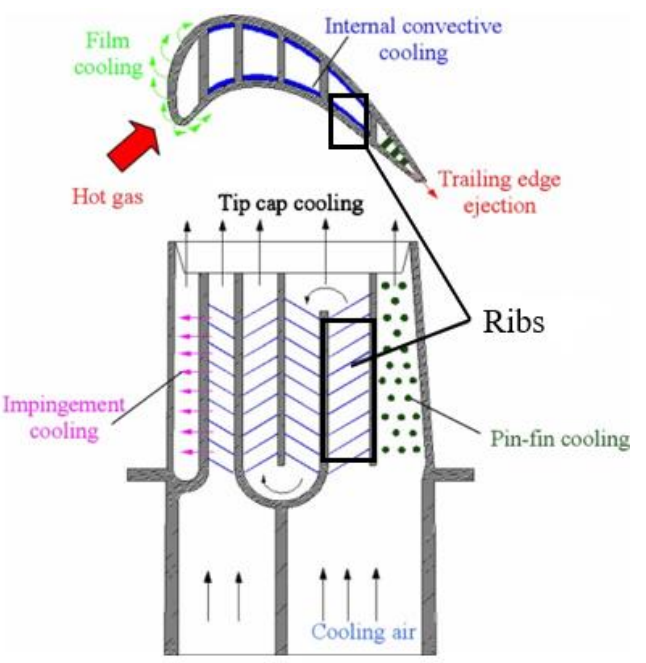

(a)

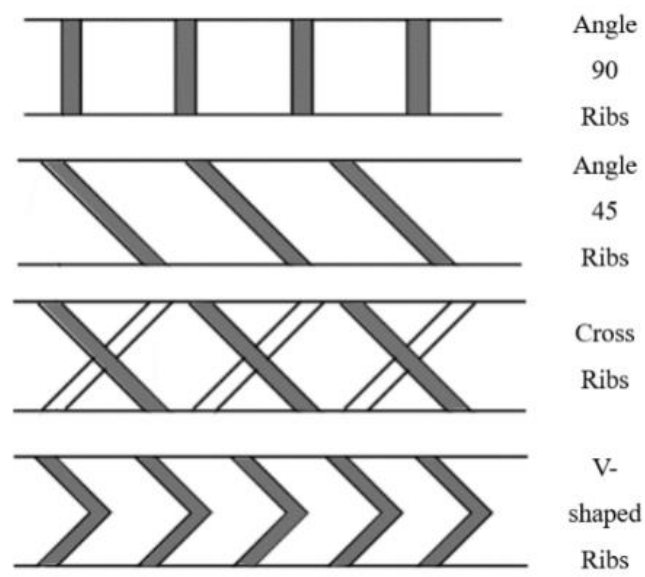

(b)

Fig. 1. The cooling details of turbine blade (a) a common arrangement of blade cooling structure (b) different rib geometries.
The determining factors for the thermal performance of the ribbed passages are rib shape, configuration, angle of attack, pitch and blockage ratio, and the parametric studies have to be done for conducting layout design of ribs in cooling channel. Moreover, since the increase in heat transfer enhancement is inevitably accompanied by the pressure loss, an optimal balance between them is widely researched. The rib-roughened surfaces were studied experimentally by Han et al. $(1978,1985,1988 a, b)$ in early years. According to the conclusions, both heat transfer and pressure loss increase with rib height. The flow field measurements indicated that ribs can destroy the viscous sublayer, generate secondary flow and incur large length scale reversed flow. In addition, when the rib pitch to height ratio is close to 10 (Han 1985), the air flow reattaches to the floor behind the recirculation zone and near the next rib. Gee and Webb (1980) provided the data about heat transfer and friction characteristics with respect to rib helix angle.

The results highlighted that the 49-degree configuration had the best overall performance. Rib is a turbulator which can enhance the turbulent intensity, it not only can produce secondary flow (or annular flow), and if is not orthogonal, it can also induce the mainstream to frequently change its direction. In studies by Han et al. (1988) the secondary flows induced by different rib configurations and their interaction had a significant effect on the heat transfer coefficient distribution inside the rectangular channel. It was concluded that the Nusselt number $(\mathrm{Nu})$ on upstream area should be higher than downstream area of the channel and the $\mathrm{Nu}$ for channels with angled ribs are generally higher than that for channels with orthogonal ribs. The friction factor $(f)$ increases as rib angle-of-attack decreasing, and the maximum occurs when the angle is $60^{\circ}$. The range of rib angle owning the highest TPF falls between $30^{\circ}$ to $40^{\circ}$.

Han et al. (1988b) found that in the 180-deg turned channel, the ribs can enhance the flow separation and secondary flow induced by inner-wall, which can improve the heat transfer. In further studies by Kiml et al. (2001), flow visualization revealed that the secondary flow firstly moves to and hits sidewall and then sharply turns 180-deg and flows back towards the opposite sidewall, which can enhance rotational momentum of the secondary flow and achieve higher averaged heat transfer. In studies by Viswanathan and Tafti (2004), the secondary flow in front of and behind ribs was clearly shown through applying the DES method.

The crossed ribs can improve the heat transfer performance by inducing secondary flow, they are also capable of making the flow return to the opposite direction. In studies about cooling channel with 45-deg crossed ribs by Jang et al. (2001), in secondary backflow region, the corner vortices markedly enhance the heat transfer, the flow moves back along the cross-stream plane and then impinges on the opposite surface. Therefore, the region near corner vortices has higher Nusselt number. In the experimental study carried out by Chang (2002), the averaged Nusselt number of duct with 45-deg crossed ribs could be elevated to 2.6 3.2 times that 
of smooth duct. The study by Abdel et al. (2004) developed the correlations of heat transfer and flow friction in the duct with unparallel ribs. The strong mean secondary flow set up in the cross-section the duce and a strong helical vortex behind the rib. Generally, the LES method can capture the flow physics more precisely. Compared to the 90-deg ribs, the friction loss of $45-\mathrm{deg}$ ribs is $60 \%$ lower under rotating operation condition in the examination carried out by Liou et al. (2004). The systematic experiments carried out by Rallabandi et al. (2009) indicated that the overall thermal performance of duct with 45-deg ribs falls as the Reynolds number increasing. On account of large blockage ratio, the taller rib height gives rise to higher pressure loss, but the overall thermal performance will decline. Multipass serpentine cooling channel with 45-deg ribs (Singh et al. 2019) and rotating two-pass channel with staggered 45-deg ribs (Deng et al. 2017) have been investigated, for increasing application of ribs or 45-deg ribs in cooling structure. Based on previous study, V-shaped ribs, as a special 45-deg ribs, was proposed and shown the higher overall thermal performance in the cooling structure. Vshaped rib was introduced and investigated using Taguchi experimental design method by Sinan Caliskan et al. (2016). And miniature V ribs arranged upstream the dimples elevate the heat transfer performance of the dimples and obtained more uniform heat transfer distribution in TLC experiment (Rao et al. 2020).

To sum up, the cooling channel with ribs is superior to smooth channel in thermal performance, and angled ribs precede the perpendicular ribs. The separation and reattachment of the shear layer are generated by rib-turbulator and secondary flow and helix flow are induced by angled ribs. 90-deg ribs don't produce the secondary flow in center of duct. For this reason, that angle ribs induce air flow, in the crossed ribs, the flow is interfered by different direction ribs. The interaction will be more different. Generally, the ribs on opposite walls are placed in parallel along the flow direction. The manufacture tolerances of blades cooling structure is hard to satisfy requirement of high precision design on account of technology restrictions (Park et al. 2016). If the opposite ribs have staggered distance, the flow pattern will be changed along the flow direction. The objective of this paper is to study the flow and heat transfer in channels with parallel ribs and crossed ribs with different staggered distances.

\section{DeSCRIPTION OF THE Problem}

\subsection{Geometry}

The investigations were performed in a straight cooling channel that equipped with $45-$ deg ribs. The different position arrangement and dislocation of crossed ribs were proposed in this paper. The inlet or outlet section is square $(W \times H=30 \times 30 \mathrm{~mm})$. The characteristic length of the channel is defined as the inlet hydraulic diameter $\left(D_{\mathrm{h}}\right)$, calculated from

$D_{h}=\frac{4 W H}{2(W+H)}$
The length of test section for heat transfer measurements is $400 \mathrm{~mm}$, and coolant inlet duct length and extra exit length are $175 \mathrm{~mm}$ and $155 \mathrm{~mm}$ respectively as illustrated by Fig. 2 (a). There were 7 ribs mounted on each primary surface, and none on the sidewalls, which is shown in Fig. 2 (b). In this study, the height $(e)$ and width $(w)$ of the ribs are set to $5 \mathrm{~mm}$, and the rib spacing $p$ is $50 \mathrm{~mm}$. The first row of ribs is $30 \mathrm{~mm}$ away from the inlet of the test section.

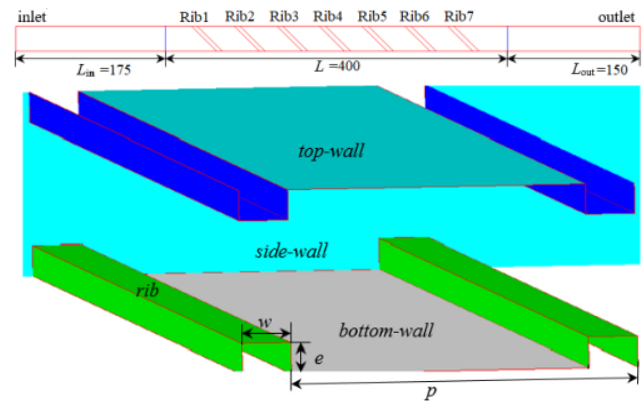

Fig. 2. Geometry definition of the cooling structure (a) dimensions of the computational domain (b) the parameters of the rib turbulator.

There are two ribbed configurations compared in this study, named as parallel (Case1) and crossed (Case2). The relative distance was analyzed for rib dislocation through only changing rib position on the bottom-wall. For case 1 , the $45^{\circ}$ ribs are placed on the opposing walls of internal cooling channel in parallel, as shown in Fig. 3(a). Figure 3(b) shows crossed $45^{\circ}$ ribs arranged in different patterns in the cooling channel. And for Case2, the top-ribs are fixed, three different relative distances between top and bottom ribs are obtained from moving the bottom-ribs downstream, corresponding to Case 2.1, Case 2.2, Case 2.3. The rib spacing $\mathrm{p}$ is also $50 \mathrm{~mm}$. The first row of bottom rib is $30 \mathrm{~mm}$ far away from inlet in Case2.1, while the distance between first bottom rib and inlet section of Case 2.2 and Case 2.3 is 45 and $60 \mathrm{~mm}$ respectively.

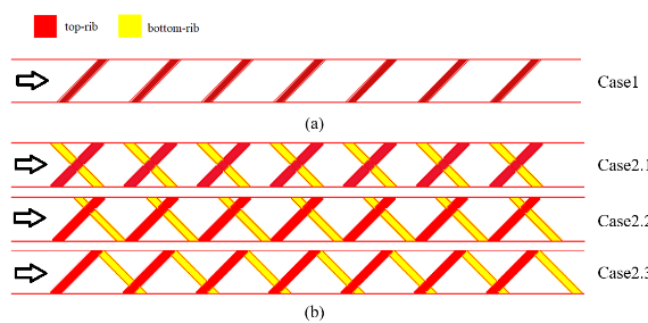

Fig. 3. Rib arrangement of (a) parallel ribs (b) crossed ribs.

\subsection{Validation of Numerical Method and Experiment}

Transient liquid crystal technology (TLCT) was applied for the heat transfer measurement of the test section. The liquid crystal changes color with temperature and the process is captured by camera. 
In the experiment, the uncertainty of narrowband liquid crystal was $\pm 0.2^{\circ} \mathrm{C}$. The technology possesses merits of full coverage, good repeatability and little interference Camci (1992).

Calculation of the heat transfer coefficient $(h)$ distribution was carried out by employing semiinfinite plate unsteady heat conduction model. Figure 4 shows the schematic of the model, and the control Eq. (2) and boundary condition Eq. (3) were listed.

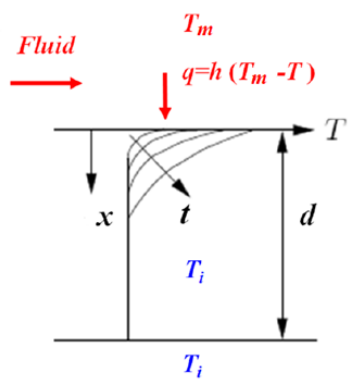

Fig. 4. Semi-infinite plate unsteady heat conduction model (Bonhoff et al. 1999).

$$
\begin{aligned}
& \frac{\partial^{2} T}{\partial x^{2}}=\frac{1}{\alpha} \frac{\partial T}{\partial t} \\
& -\lambda(\partial T / \partial x)=h\left(T_{m}-T_{w}\right)
\end{aligned}
$$

Where $\lambda$ and $\alpha$ are material conductivity and expansion coefficient respectively. $T_{\mathrm{m}}$ represents the local mainstream temperature in cooling channel, and $T_{\mathrm{w}}$ is the temperature of heat transfer plate. $h$ is the heat transfer coefficient of the local surface.

$\frac{T_{w}-T_{i}}{T_{m}-T_{i}}=1-\exp \left(\frac{h^{2} \alpha t}{\lambda^{2}}\right) \operatorname{erfc}\left(\frac{h \sqrt{\alpha t}}{\lambda}\right)$

$T_{\mathrm{i}}$ is the initial wall temperature, which is equal to environmental temperature in this study. The whole surface temperature $\left(T_{\mathrm{w}}\right)$ was obtained by processing photo data in TLCT, the local $T_{\mathrm{w}}$ corresponding to local pixel color will be applied in the program solving heat transfer coefficient.

The analytical solution of Eq. (4) was worked out to obtain $h$ by means of computer programs (Ekkad and Han 2007). The parameters measured in experiment, including $T_{\mathrm{w}}, T_{\mathrm{i}}, T_{\mathrm{m}}$, and other essential condition values were input in software program.

As shown in Fig. 5. an experimental facility was constructed to obtain heat transfer and friction loss data. The centrifugal fan provided steady air mass flow condition and wire mesh heater generated $60 \sim 80^{\circ} \mathrm{C}$ hot air to heat the test section.

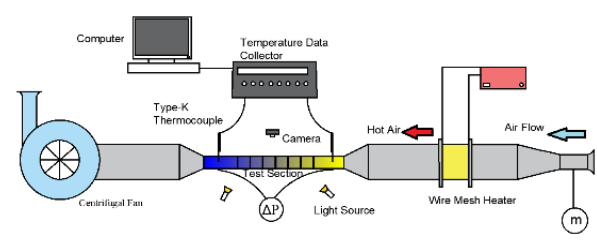

Fig. 5. Experimental facility.
$T_{\mathrm{m}}$ was measured by Type-K thermocouples with $\pm 0.2^{\circ} \mathrm{C}$ uncertainty placed at the inlet and outlet of the test section. Meanwhile, data was gathered by temperature data collector. Pressure measuring equipment obtained the total pressure drop of the test section and mass flow rate in the entrance. The uncertainty of the TSI was $\pm 1 \mathrm{~Pa}$. Based on the method proposed by Moffat (1988), the maximum uncertainty of $R e$ and $h$ were calculated at approximately $4 \%$ and $9 \%$.

A darkroom was built for cutting off ambient light and reducing interference. The heat transfer surface color change was caught by camera. All data including thermal images, temperature and pressure were conveyed to the computer and transformed to desired quantities.

For validation of numerical method, test section in the experiments only included 90-deg parallel ribbed channel and 45-deg parallel ribbed channel. The geometric parameters of rib and channel were consistent except rib angle.

The steady method was performed in this paper. The RANS (Reynolds-Averaged Navier-Stokes) approach is primarily used to solve turbulent flow. And it is significant to choose a reasonable turbulence model for assuring numerical accuracy.

In this paper, enhanced heat transfer coefficient $(\mathrm{Nu} / \mathrm{Nu})$ and normalized resistance coefficient $\left(f / f_{0}\right)$ were respectively introduced into heat transfer and friction loss assessment.

The Nusselt number $(\mathrm{Nu})$ reveals the heat transfer performance of the cooling channels, and is defined as follow:

$N u=\frac{h D_{h}}{\lambda}$

Where $h$ is heat transfer coefficient, $D_{\mathrm{h}}$ is hydraulic diameter, $\lambda$ is air thermal conductivity.

$N u_{0}$ is the averaged Nusselt number of the smooth channel, whose correlation proposed by Gnielinski (1976) is selected as

$$
\begin{aligned}
& N u_{0}=0.0214\left(\operatorname{Re}^{0.8}-100\right) \operatorname{Pr}^{0.4} \\
& \left(0.5 \leq \operatorname{Pr} \leq 1.5,10^{4} \leq \operatorname{Re} \leq 5 \times 10^{6}\right)
\end{aligned}
$$

Where, $R e$ is defined as follow:

$$
R e=\frac{V_{\text {in }} D_{\mathrm{h}}}{v}
$$

Where $v$ is the kinematic viscosity, $V_{\text {in }}$ is the velocity of the inlet. It is easier to describe the friction loss by introducing a non-dimensional quantity, Fanning friction factor $(f)$ (Liu et al. 2015), calculated from

$f=\frac{\Delta P}{\frac{1}{2} \rho u^{2}} \frac{D_{h}}{4 L}$

Where $\Delta P$ is total pressure drop across the test section.

Similarly, $f_{0}$ is the friction coefficient of a smooth channel defined by Petukhov et al. (1970): 


$$
\begin{aligned}
& f_{0}=2(2.236 \ln R e-4.639) \\
& \left(10^{4} \leq \operatorname{Re} \leq 5 \times 10^{6}\right)
\end{aligned}
$$

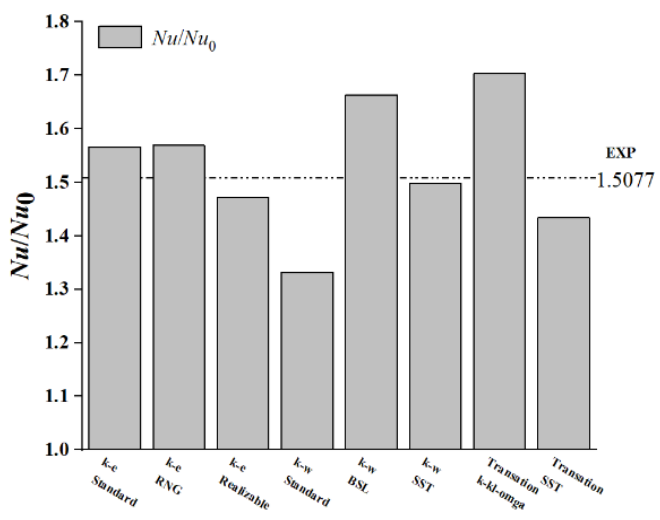

(a)

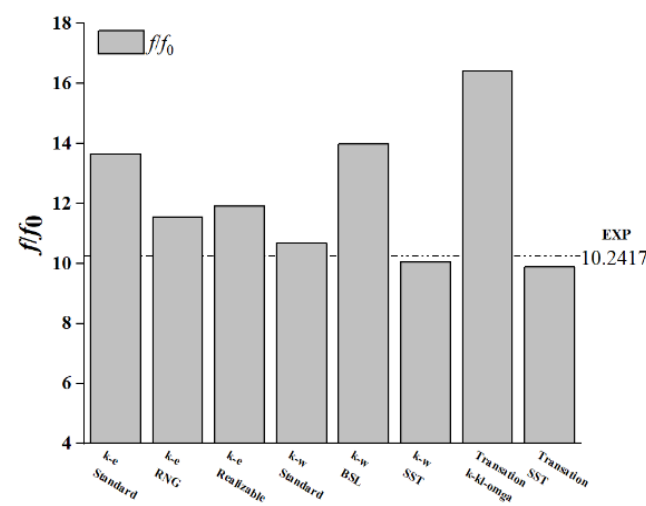

(b)

Fig. 6. (a) $N u / N u_{0}(b) f / f_{0}$ of 90 -deg ribbede channel solved by different turbulence models and obtained from experiment at $R e=30529$.

The $N u / N u_{0}$ and $f l f_{0}$ of the cooling channel with 90-deg ribs are shown in Fig. 6. The SST $k$ - $\omega$ model is closest to experimental results and was selected for CFD.

In existing researches it is common to obtain the same conclusion that SST $k$ - $\omega$ model has higher precision to predict the turbulent flow in the channel with ribs (Lu and Jiang 2006). SST $k$ - $\omega$ model was proposed by Menter $(1992,1994)$, the equations of Reynolds stress and $k$ used in the $k$ - $\omega$ model proposed by Wilcox $(1993,1994)$, are Eqs. (10) and (11) respectively. But beyond that, Eq. (12) was added, using equation $\varepsilon=k \omega$, in order to transform $\varepsilon$ equation into $\omega$ equation.

$\tau_{i j}=-\rho \overline{u_{i}{ }^{\prime} u_{j}{ }^{\prime}}=\mu_{t}\left(\frac{\partial u_{i}}{\partial x_{j}}+\frac{\partial u_{j}}{\partial x_{i}}\right)-\frac{2}{3} \rho k \delta_{i j}$

$$
\begin{aligned}
& \frac{\partial(\rho k)}{\partial t}+\operatorname{div}(\rho k \vec{u})= \\
& \operatorname{div}\left[\left(\mu+\frac{\mu_{t}}{\sigma_{k}}\right) \operatorname{grad} k\right]+P_{k}-\beta^{*} \rho k \omega \\
& \frac{\partial(\rho \omega)}{\partial t}+\operatorname{div}(\rho \omega \vec{u})= \\
& \operatorname{div}\left[\left(\mu+\frac{\mu_{t}}{\sigma_{\omega, 1}}\right) \operatorname{grad} \omega\right]+ \\
& \gamma_{2}\left(2 \rho S_{i j} \cdot S_{i j}-\frac{2}{3} \rho \omega \frac{\partial u_{i}}{\partial x_{j}} \delta_{i j}\right)- \\
& \beta_{2} \rho \omega^{2}+2 \frac{\rho}{\sigma_{\omega, 2}} \frac{\partial k}{\partial x_{k}} \frac{\partial \omega}{\partial x_{k}}
\end{aligned}
$$

In the investigation, the results of experimental with error bar and results of numerical predictions using SST $k-\omega$ model are compared in Fig. 7. The SST $k-\omega$ model slightly over-predicted the heat transfer but under-predicted the friction loss. Nu/Nu is descending and $f / f_{0}$ is increasing with $R e$ increasing in the 45-deg ribbed cooling channel. As for the 90deg ribbed channel, $N u / N u_{0}$ is $34.68 \%$ less than and $f l f_{0}$ is $38.44 \%$ less than that of $45-\mathrm{deg}$ ribbed channel and $\mathrm{Nu} / \mathrm{Nu}$ mostly keeps constant with the increase of Re. In Fig.7(c), it was more obvious that the largest $N u$ values appeared in the front of each rib in the experiment result. The level of heat transfer enhancement was the biggest just upstream the Rib2 as the result of the impingement effect (1). On account of the reverse flow in the back end of rib, the $\mathrm{Nu}$ value was relatively smaller. The small symmetrical vortexes gave rise to local heat transfer enhancement (2)). The most central area among the ribs presented a higher heat transfer level caused by the reattachment of separated air.

\subsection{Mesh and Independence Verification}

High quality multi-block structured mesh was generated to model the fluid flow for both parallel and crossed cases, as shown in Fig. 8. Mesh was refined near the endwalls to resolve the turbulent boundary layer. Y+ value was kept at the order of unity.

Appropriate mesh density is vital to accurately model fluid dynamics and heat transfer with relatively high computational efficiency, so the mesh independence verification was carried out. In this study, three mesh configurations were compared for the cooling channel with parallel ribs, viz. Mesh1(2.21million), Mesh2(2.88million), Mesh3(3.23million). The simulation results of normalized Nusselt number at $R e=21587$ are listed in Table1. It can be seen that the value of $\mathrm{Nu} / \mathrm{Nu} u_{0}$ has smaller distinction between Mesh2 and Mesh3. Moreover, Fig. 9 presents the $\mathrm{Nu} / \mathrm{Nu} \mathrm{u}_{0}$ distribution between Rib1 and Rib2 in $x$ direction. 


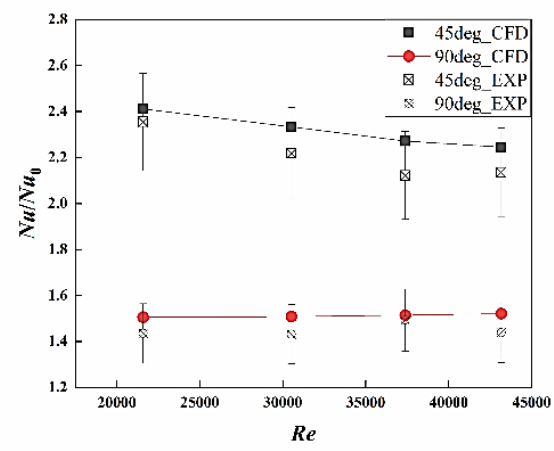

(a)

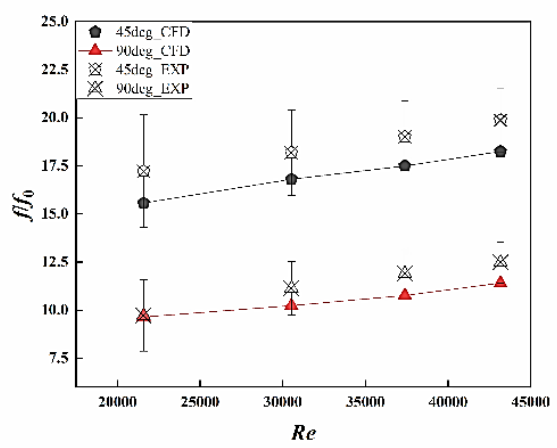

(b)
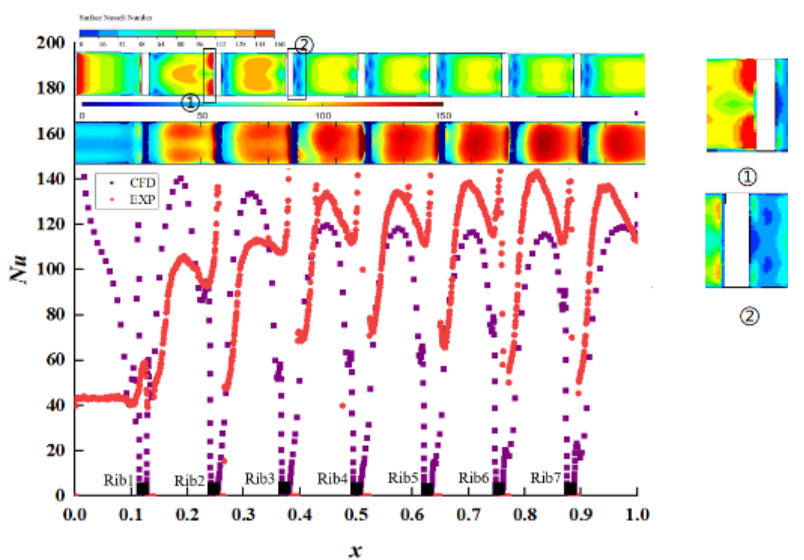

(2)

(c)

Fig. 7. Evolution of CFD and experimental results with $R e$ (a) $\mathrm{Nu} / \mathrm{Nu} u_{0}$ (b) $f / f_{0}$ (c) distribution of $\mathrm{Nu}$.
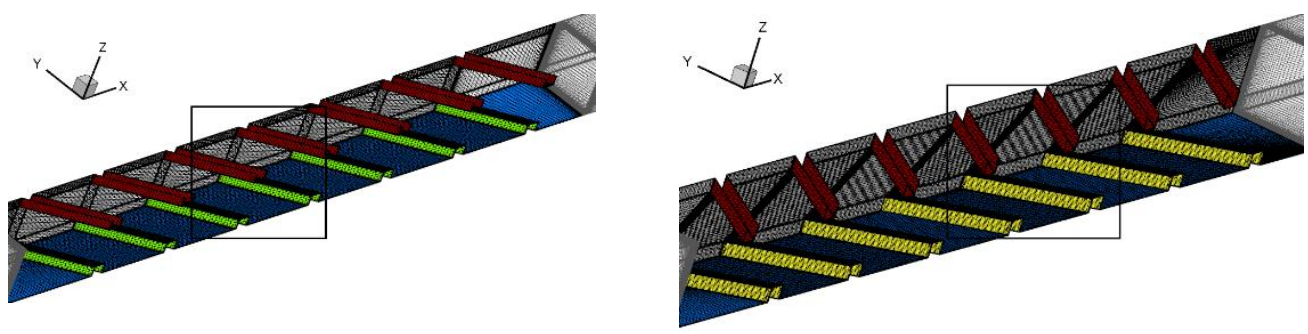

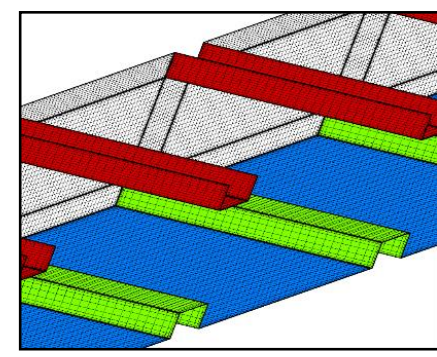

(a)

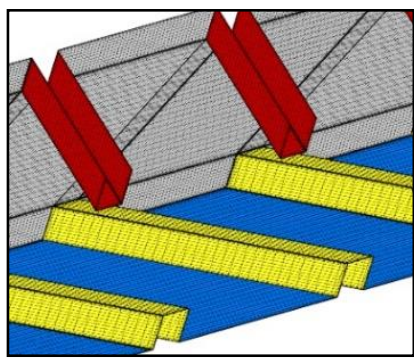

(b)

Fig. 8. Computational meshes (a) Case1

(b) Case2.

The heat transfer characteristics obtained using Mesh2 and Mesh3 are similar. Consequently, Mesh2 was selected as the computational mesh. The mesh independence validation of the test sections with crossed ribs was conducted by the same method and the desired mesh node number was at the same level, so Mesh2 was also suitable for Case2. 
Table.1 Normalized Nusselt number calculated using different mesh configurations.

\begin{tabular}{|c|c|}
\hline number of nodes & $N u / N u_{0}$ \\
\hline Mesh1 & 2.491 \\
\hline Mesh2 & 2.419 \\
\hline Mesh3 & 2.425 \\
\hline
\end{tabular}

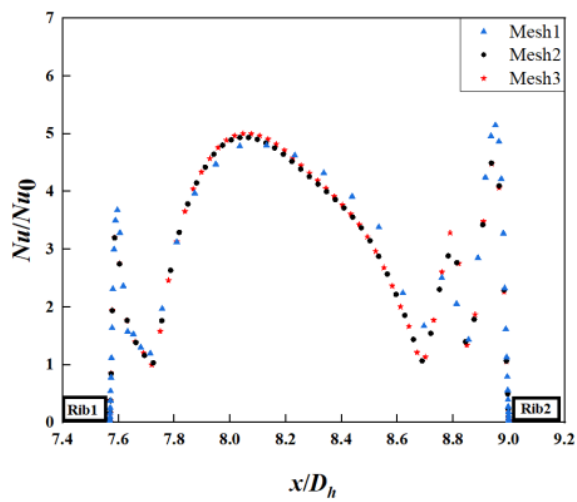

Fig. 9. $\mathrm{Nu} / \mathrm{Nu} u_{0}$ distribution between Rib1 and Rib2 in $x$ direction at $R e=21587$.

\subsection{Configuration and Boundary Condition}

The numerical steady solution was carried out on the commercial CFD platform. The velocity and pressure coupling scheme was SIMPLEC algorithm. The second order upwind scheme was adopted for the spatial discretization of continuity, momentum and energy equations. The convergence criteria for the continuity equation were residual reaches below $10-4$ and the inlet total pressure becomes steady.

In this study, the coolant air was considered as compressible ideal gas. The temperature difference of the computational domain was small, ranging from $293 \mathrm{~K}$ to $323 \mathrm{~K}$. Therefore, the specific heat capacity and the air thermal conductivity coefficient were set as constant, i.e., $C_{\mathrm{p}}=1006.43 \mathrm{~J} /(\mathrm{kg} \cdot \mathrm{K})$ and $\lambda=0.0242 \mathrm{~W} /(\mathrm{m} \cdot \mathrm{K})$. And the Sutherland law was selected to describe viscosity. The cooled walls including side-wall, top-wall and bottom-wall were isothermal wall whose temperature was $323 \mathrm{~K}$ and the extend walls outside the test section were specified as adiabatic wall. All the endwalls were specified as no-slip boundary. The inlet mass flow rate condition was specified according to the Reynolds number, whose value varied between 21587 and 43174.

\section{RESULTS AND DISCUSSION}

\subsection{Friction and Heat Transfer}

The introduction of ribs not only can enhance heat transfer, but also leads to the increase in blockage ratio. The air coolant came from compressor will make flow losses in the cooling channel, which is described by the normalized resistance coefficient $\left(f / f_{0}\right)$. The numerical result is shown in Figs. 10-13, including $f / f_{0}$ and $\mathrm{Nu} / \mathrm{Nu} u_{0}$. In Fig.10, $f / f_{0}$ increases with the Reynolds number, this is because the increase of turbulence intensity brings more friction and impact. Compared to the crossed rib cases (Case 2), the pressure loss in the parallel rib case (Case 1) is more serious with $25.4 \%$ higher.

As for the three crossed rib arrangements with different staggered distances in Case 2, their normalized resistance coefficients are very close. $f / f_{0}$ of Case 2.2 is the largest and that of Case 2.3 is the smallest. In conclusion, the crossed arrangement of ribs can reduce the pressure loss and there should be an optimal staggered distance with which the pressure loss is the smallest. Among the current three configurations in Case 2, Case 2.3 is the best for controlling the pressure loss.

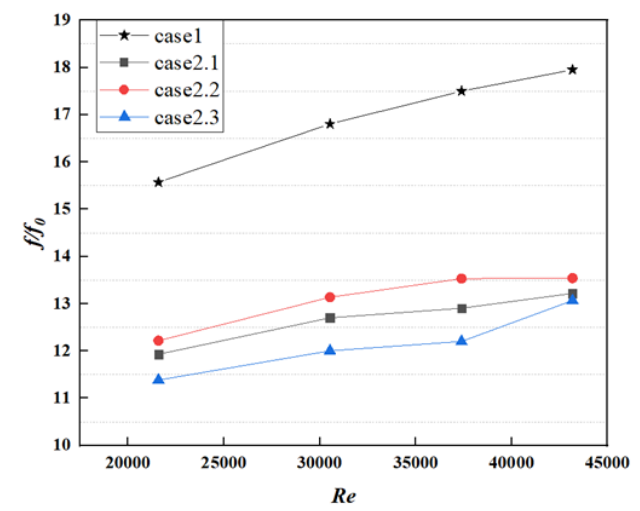

Fig. 10. Normalized resistance coefficient $\left(f / f_{0}\right)$ of all cases versus inlet $R e$.

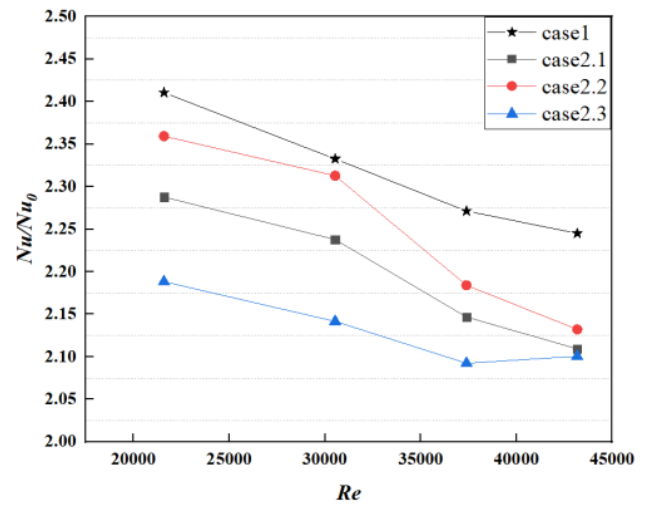

Fig. 11. Normalized Nusselt number plotted with inlet $R e$.

As shown in Fig. 11., normalized Nusselt number presents decreasing trend for all the cases as the Reynolds number increasing. The heat transfer enhancement of Case 1 is slightly higher than Case 2 , but its pressure loss is much larger. Similarly, the heat transfer performance of Case 2.3 is the worst among all the cases, which is also closely matched with friction characteristics. Generally, with the heat transfer enhanced, the friction would simultaneously increase (Han, 1978, 1984, 1985). The ideal cooling structure possesses high heat transfer capacity and relatively low friction penalty at the same time.

The overall performance difference derives from the promotion of heat transfer by specific structural characteristics, such as the front corner of inclined 
ribs depicted in Fig. 12. The heat transfer distribution was solved under $R e=21587$. All the strongest heat transfer regions are located in the front corner, while the weakest are in the back corner. And the leading edge of ribs also have higher heat transfer coefficient, which is not shown in Fig. 12. And part of the sidewall in the back corner has stronger heat transfer resulting from the airflow impingement.

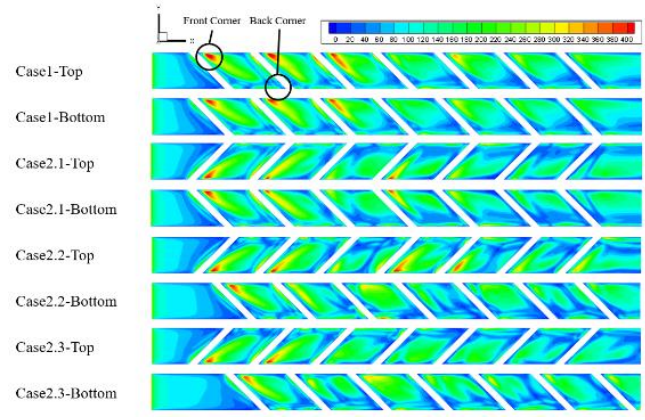

Fig. 12. $N u$ distribution on the top and bottom wall at $R e=21587$.

In Case 1, since the parallel rib arrangement is symmetrical, the top and bottom wall have identical heat transfer distribution. The first crossed rib arrangement (Case 2.1) and its heat transfer distribution are centrosymmetric. $N u$ becomes smaller downstream of the second rib row in $x$ direction.

However, the phenomenon that higher heat transfer is concentrated at the front corner still exists due to the secondary helical flow. When the ribs are staggered in $x$ direction, heat transfer distribution of both walls becomes obviously different in Case 2.2 and Case 2.3 and their heat transfer situation is also much different from each other due to different staggered distances. Therefore, the crossed rib arrangement has great impact on the heat transfer distribution of cooling structure and then the temperature distribution of turbine blades. The front corner is still the strongest heat transfer area. Overall, Case 1 presents the best heat transfer performance, followed by Case 2.2, Case 2.1 and Case 2.3.

Figure 13 presents the variation of $\mathrm{Nu} / \mathrm{Nu}$ along the centerline of both primary surfaces. For Case1, $\mathrm{Nu} / \mathrm{Nu}$ o generally keeps growing behind the inlet section and the peak appears just downstream of Rib3. In the downstream area, $N u / N u_{0}$ declines gradually in general. Case 2.1 is similar with Case 1 in $N u / N u_{0}$ distribution, but the greatest value appears between Rib2 and Rib3. Due to the ribs were staggered, the averaged $\mathrm{Nu} / \mathrm{Nu} u_{0}$ on the topwall is $2.46 \%$ larger than on the bottom-wall in Case 2.2. And the averaged $\mathrm{Nu} / \mathrm{Nu} u_{0}$ on the top-wall is $0.36 \%$ lower than on the bottom-wall in Case 2.3. However, Case 2.2 shows the highest heat transfer performance in Case 2, and its heat transfer distribution is more disorganized.

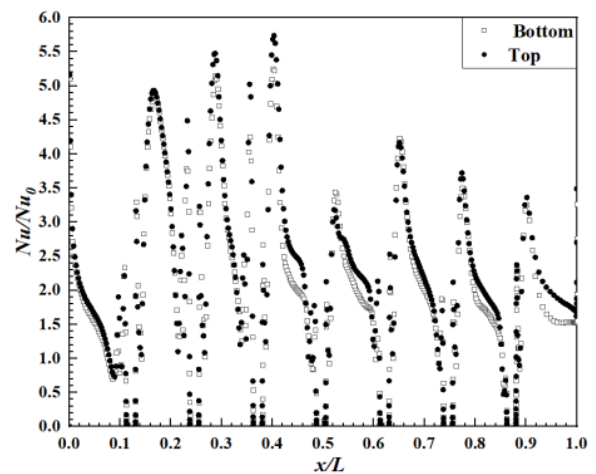

(a)

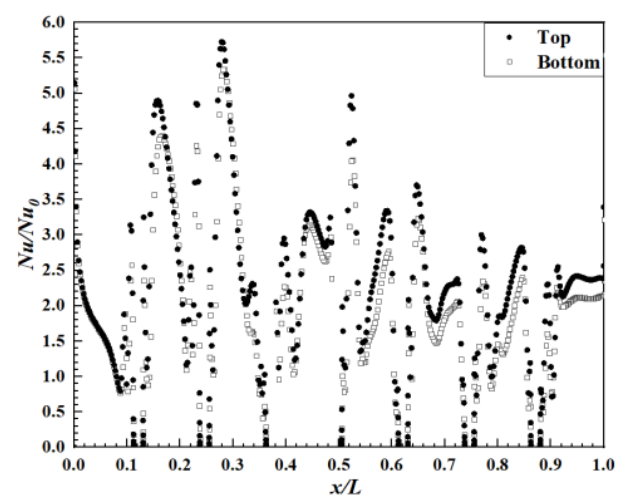

(b)

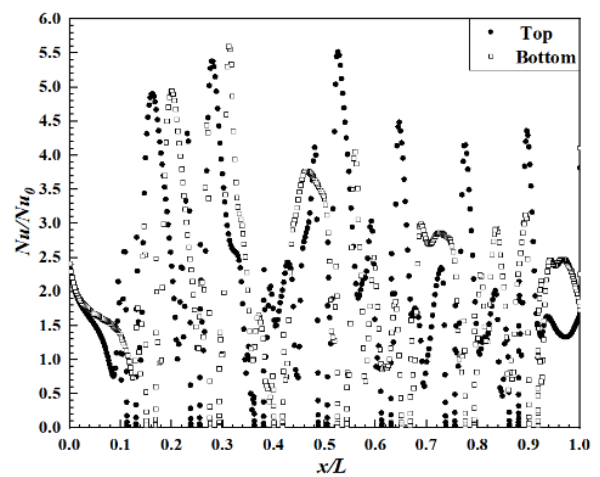

(c)

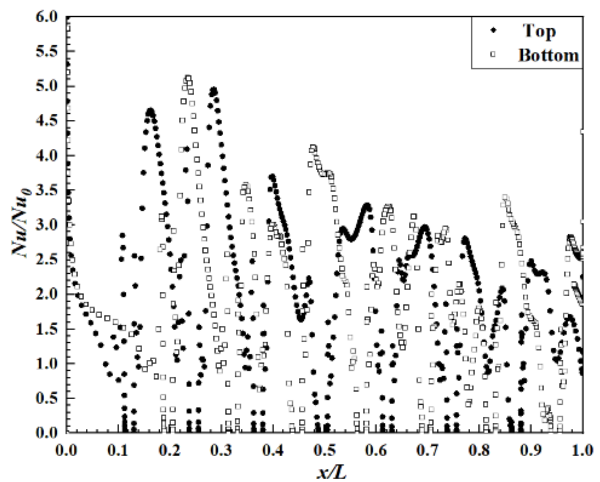

(d)

Fig. 13. Normalized Nusselt number $(\mathrm{Nu} / \mathrm{Nu}))$ along the centerline of the top-wall and bottomwall at $R e=21587$. 


\subsection{Flow and Heat Transfer Details}

All heat transfer phenomena are correlated with the flow field characteristics. In this section, the relationship between fluid flow and heat transfer details will be discussed. Figure 14. shows the turbulent kinetic energy contours and superimposed streamlines at $x-y$ plane between Rib1 and Rib4, with half of the rib height offset from the bottom wall.

The airflow approaches and separates at the rib leading edge, which results in the backflow and the helical vortex in three dimensions. The helical vortices mostly appear just downstream of the ribs, as shown in Fig. 14. The streamlines indicate the transport of airflow from the front corner towards the back corner in all cases. The small helical flow behind the ribs can enhance heat transfer, so the Nusselt number in the front corner behind the ribs is relatively high.

The secondary flow along the ribs impinges to the opposite sidewall and gathers at the back corner, which is comparable to the flow characteristic in the expand nozzle. In Case 1, the backflow vortex is stronger and the turbulent kinetic energy (TKE) is generally higher than Case 2, resulting in more friction loss. Due to the staggered ribs, lower levels of TKE and smoother streamlines are observed in Case 2.2 and Case 2.3.

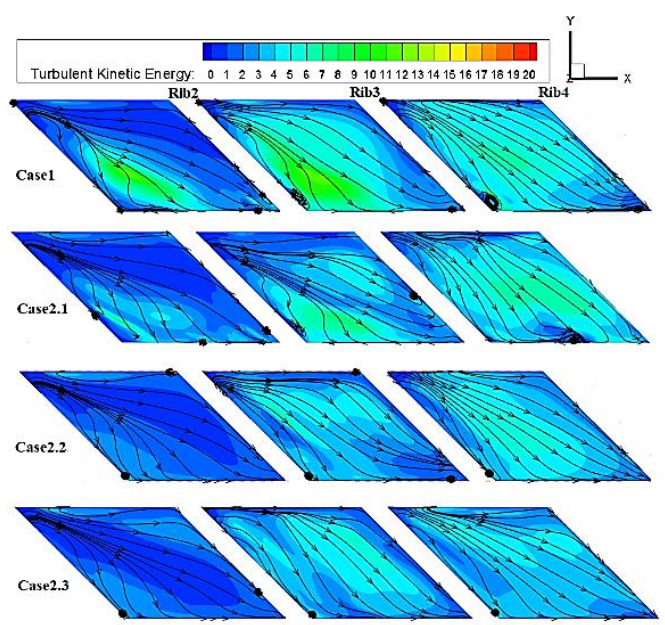

Fig. 14. Streamlines and TKE distribution of all cases between Rib1 and Rib4 at $R e=21587$.

The transverse views of turbulent kinetic energy and streamlines in the middle position of upper ribs have been depicted in Fig. 15. It can be seen that the structure of the longitudinal vortices is frequently transformed because of the rib turbulators. And the turbulent kinetic energy gradually increases with the development of the fluid flow and the maxima are mostly generated in the center of vortices.

The transverse flow distribution is symmetrical in Case1 and Case2.1. The secondary flow is divided to upper and lower regions with counter-rotating vortices in them for Case1. In case1, the small helical vortices form behind the ribs and the large scale counter-rotating vortices occupy the center of the channel. The streamlines in Case 1 can also clearly reflect that the airflow is transported from one sidewall to the opposed sidewall along the ribs continuously. It can be found that in Case 2, the pair of large scale counter-rotating secondary vortices is rotating as the flow moving downstream. Moreover, it is a prominent feature that the transverse secondary flow is generally anticlockwise for Case 2 .

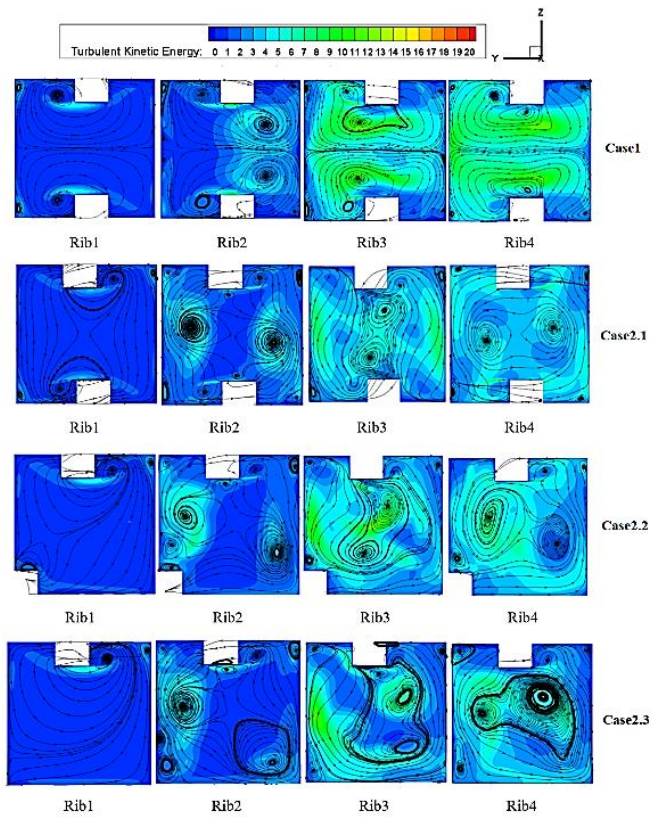

Fig. 15. TKE distribution and streamlines plotted at $y-z$ plane.

On account of the staggered rib arrangement in Case2.2 and Case2.3, the flow field presents asymmetric and uneven features. However, Case2.2 and Case2.3 have some common flow features with Case2.1. For example, the pair of large scale counterrotating vortices still exists and keeps rotating as moving downstream, but their sizes are different from each other. The chaotic flow phenomena lead to scattered $\mathrm{Nu}$ distribution. Case2.3 has the lowest level of TKE, and also has the lowest heat transfer enhancement as well as friction loss.

In Fig. 16, the helical vortices in the separation region behind the ribs and the formation of the large scale vortices in the center of the channel for Case2.1 have been schematically illustrated. It can be found that the mainstream flows across and separates from the ribs on the bottom-wall.

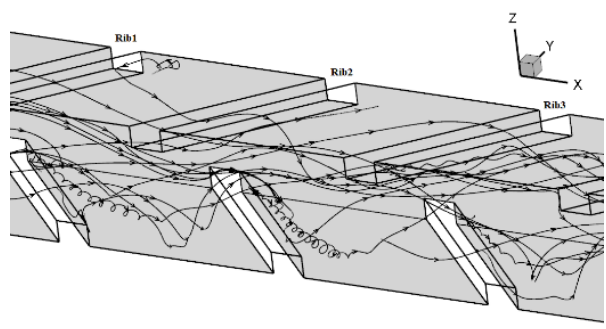

Fig. 16. Volume streamlines of Case2.1 at $R e=21587$. 
The helical vortex always begins from the front corner and creates a separation bubble. And then the helical vortex is forced to flow along the 45-deg rib towards the sidewall. Soon afterwards, the flow sharply turns 180-deg and flows back towards the opposite sidewall, which results in a development of the transverse secondary flow in form of counterrotating large vortices. Meanwhile, the small helical eddies mainly exist behind the ribs and near the sidewalls.

The TKE distributions at the channel centerline are depicted in Fig. 17. It is obvious that the TKE of crossed rib configurations (Case2) is smaller than parallel rib configuration (Case1) and Case2.3 has the lowest TKE level among Case2. Therefore, the parallel rib configuration has higher heat transfer enhancement and friction loss than the crossed rib configuration, and the heat transfer or friction loss of the crossed rib configuration would further decrease if using inappropriate staggered arrangement. Averaged TKE of the flow pronouncedly rises downstream of Rib2. And the maxima of the TKE are distributed in the area of helical vortices and separated shear layer, hence causes increase in nearwall heat transfer. Nevertheless, the chaotic interaction between the rib-induced secondary flows and the core coolant flow also bring much pressure loss due to viscous friction. How to control the turbulence is crucial in the process of designing ribs.

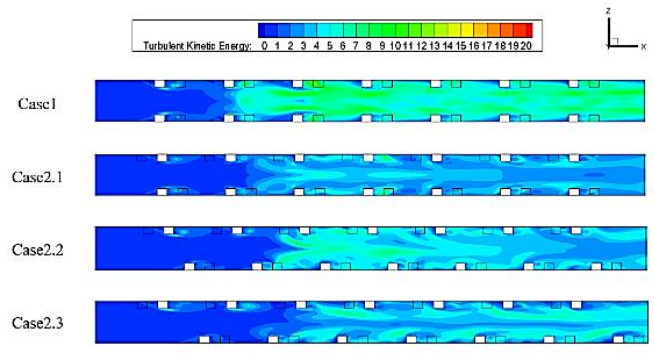

Fig. 17. Turbulent kinetic energy (TKE) contours at $x-z$ plane for all cases

\subsection{Overall Thermal Performance}

In previous studies, $N u / N u_{0}$ was used to measure heat transfer enhancement taking the place of $S t / S t_{0}$ (Gee 1980 and Han 1985). In this paper, overall consideration of heat transfer and friction loss is significant and the thermal performance factor $(T P F)$ is used to balance both factors (Tanda 2011). The $T P F$ is defined as follows.

$$
T P F=\frac{N u / N u_{0}}{\left(f / f_{0}\right)^{\frac{1}{3}}}
$$

$N u / N u_{0}$ and $f / f_{0}$ are applied to illustrate the promotion of heat transfer and pressure loss separately. TPF integrates the both factors according to the level of significance. However, the heat transfer has a greater effect than the friction loss.

It can be concluded from Fig. 18. that $T P F$ decreases with the Reynolds number increasing. TPF of Case

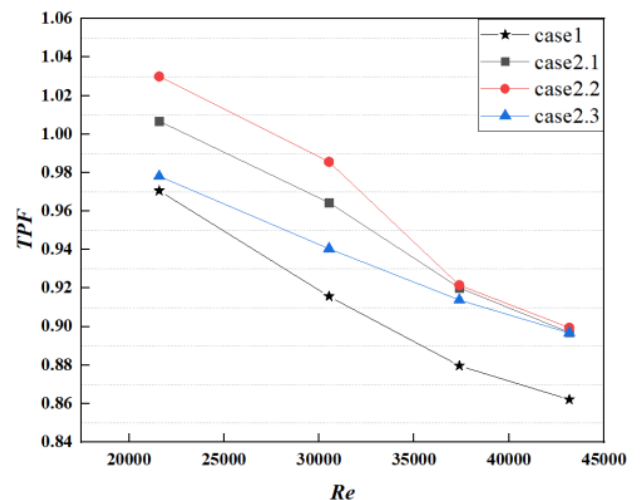

Fig. 18. Thermal performance factor against inlet $\boldsymbol{R e}$.

2.2 is the largest for the range of $R e$ investigated and that of Case 2.1 is the second largest. The Case1 has the worst overall thermal performance. And averaged TPF under different $R e$ conditions of Case 2.2 is $5.7 \%$ higher than Case 1 . Therefore, the penalty of pressure loss is relatively high compared to the gain from heat transfer enhancement for Case1. As mentioned before, high near-wall TKE is beneficial to the heat transfer since the turbulence accelerates the heat exchange between the coolant and the walls. Meanwhile, turbulence will also enhance inner TKE far away from the wall which gives rise to significant pressure loss. $T P F$ is an important performance indicator to guide the cooling channel design of the turbine blade. Overall, for parallel and crossed 45-deg rib configurations, the overall thermal performance of crossed rib configuration is better and it is more beneficial to properly stagger the opposite ribs.

\section{CONClusions}

Flow and heat transfer performance of 45-deg rib configurations were studied in this paper using numerical simulation method. The flow characteristics and heat transfer distribution have shown close association. So, the related conclusions have been drawn.

1. Compared with experimental data, SST $k$ - $\omega$ model has higher precision to predict the turbulent flow in the channel with ribs than other turbulence models.

2. Averaged $f l f_{0}$ of 45 -deg parallel rib configuration is $25.4 \%$ higher than that of 45 -deg crossed rib configuration.

3. The angled ribs can induce the secondary flow and generate small helical vortices at front corner behind the ribs. The large scale counterrotating vortices occupy the center of the channel.

4. The 45-deg parallel rib configuration has the highest heat transfer enhancement as well as friction loss.

5. At $R e=21587$, the averaged turbulent kinetic energy of Case 2.2 is $22.4 \%$ lower than Case1. Based on the inline crossed rib configuration, 
different staggered distances between theopposite ribs contribute to different turbulent kinetic energy variations.

6. The overall thermal performance of 45-deg crossed rib configurations is superior to parallel rib configuration. The overall thermal performance of Case 2.2 is the best for the range of $R e$ investigated.

7. 45-deg crossed rib configuration with proper dislocation is capable of improve overall thermal performance through adjusting the flow state. Hence it can meet the heat transfer requirement of the cooling system with relatively lower pressure loss.

\section{ACKNOWLEDGEMENTS}

This work is supported by National Science and Technology Major Project (2017-V-0012-0064), also supported by the National Natural Science Foundation of China (NSFC) [No. 51509052].

\section{REFERENCES}

Abdel-Wahab, S. and D. K. Tafti (2004, January). Large eddy simulation of flow and heat transfer in a staggered 45 ribbed duct. In Turbo Expo: Power for Land, Sea, and Air 41685, 723-734.

Bonhoff, B., S. Parneix, J. Leusch, B. V. Johnson, J. Schabacker and A. Bölcs (1999). Experimental and numerical study of developed flow and heat transfer in coolant channels with 45 degree ribs. International Journal of Heat and Fluid Flow 20(3), 311-319.

Caliskan, S., M. Nasiri Khalaji, S. Baskaya and I. Kotcioglu (2016). Design analysis of impinging jet array heat transfer from a surface with V-shaped and convergent-divergent ribs by the Taguchi method. Heat Transfer Engineering 37(15), 1252-1266.

Camci, C., K. Kim, and S. A. Hippensteele (1992). A new hue capturing technique for the quantitative interpretation of liquid crystal images used in convective heat transfer studies.

Deng, H., Y. Li, Z. Tao, G. Xu, and S. Tian (2017). Pressure drop and heat transfer performance in a rotating two-pass channel with staggered 45deg ribs. International Journal of Heat and Mass Transfer 108, 2273-2282.

Ekkad, S. V., and J. C. Han (2000). A transient liquid crystal thermography technique for gas turbine heat transfer measurements. Measurement Science and Technology 11(7), 957.

Gee, D. L., and R. L. Webb (1980). Forced convection heat transfer in helically ribroughened tubes. International Journal of Heat and Mass Transfer 23(8), 1127-1136.

Gnielinski, V. (1976). New equations for heat and mass transfer in turbulent pipe and channel flow. International Journal of Chemical Engineering 16(2), 359-368.

Han, J. C. and A. Rallabandi (2010). Turbine blade film cooling using PSP technique. Frontiers in Heat and Mass Transfer (FHMT) 1(1).

Han, J. C., L. R. Glicksman and W. M. Rohsenow (1978). An investigation of heat transfer and friction for rib-roughened surfaces. International Journal of Heat and Mass Transfer 21(8), 1143-1156.

Han, J. C. (1984). Heat transfer and friction in channels with two opposite rib-roughened walls. Journal of Heat Transfer 106(4): 774781.

Han, J. C., J. S. Park and C. K. Lei (1985). Heat transfer enhancement in channels with turbulence promoters. Journal of Engineering for Gas Turbines and Power 107(3), 628-635.

Han, J. C. and J. S. Park (1988a). Developing heat transfer in rectangular channels with rib turbulators. International journal of heat and mass transfer 31(1), 183-195.

Han, J. C., P. R. Chandra and S. C. Lau (1988). Local heat/mass transfer distributions around sharp $180 \mathrm{deg}$ turns in two-pass smooth and ribroughened channels. Journal of Heat Transfer 110(1), 91-98.

Jang, Y. J., H. C. Chen and J. C. Han (2001). Flow and heat transfer in a rotating square channel with 45 deg angled ribs by Reynolds stress turbulence model. Journal of Turbomachinery 123(1), 124-132.

Kiml, R., S. Mochizuki and A. Murata (2001). Effects of rib arrangements on heat transfer and flow behavior in a rectangular ribroughened passage: application to cooling of gas turbine blade trailing edge. Heat Transfer 123(4), 675-681.

Liou, T. M. and G. Y. Dai (2004). Pressure and flow characteristics in a rotating two-pass square duct with 45-deg angled ribs. Journal of Turbomachinery 126(1), 212-219.

Liu, J., Y. Song, G. Xie and B. Sunden (2015). Numerical modeling flow and heat transfer in dimpled cooling channels with secondary hemispherical protrusions. Energy 79, 1-19.

Lu, B. and P. X. Jiang (2006). Experimental and numerical investigation of convection heat transfer in a rectangular channel with angled ribs. Experimental Thermal and Fluid Science 30(6), 513-521.

Menter, F. R. (1992). Performance of popular turbulence model for attached and separated adverse pressure gradient flows. AIAA journal 30(8), 2066-2072.

Menter, F. R. (1994). Two-equation eddy-viscosity turbulence models for engineering applications. AIAA journal 32(8), 1598-1605. 
Z. Wang et al. / JAFM, Vol. 14, No. 5, pp. 1535-1546, 2021.

Wilcox, D. C. (1993). Comparison of two-equation turbulence models for boundary layers with pressure gradient. AIAA Journal 31(8), 14141421.

Wilcox, D. C. (1994). Simulation of transition with a two-equation turbulence model. AIAA Journal 32(2), 247-255.

Moffat, R. J. (1988). Describing the uncertainties in experimental results. Experimental Thermal and Fluid Science 1(1), 3-17.

Park, S., H. Moon, H. Chung, J. S. Park and H. H. Cho (2019). Effect of Manufacturing Tolerances on the Cooling Performance of Internal Rib Turbulated Passages. Heat Transfer Engineering 40(5-6), 418-428.

Petukhov, B. S., T. F. Irvine and J. P. Hartnett (1970). Advances in heat transfer. Academic, New York 6, 503-564.

Rallabandi, A. P., H. Yang and J. C. Han (2009). Heat transfer and pressure drop correlations for square channels with $45 \mathrm{deg}$ ribs at high
Reynolds numbers. Journal of Heat Transfer 131(7).

Rao, Y. and P. Zhang (2020). Experimental study of heat transfer and pressure loss in channels with miniature V rib-dimple hybrid structure. Heat Transfer Engineering 41(15-16), 1431-1441.

Singh, P., Y. Ji and S. V. Ekkad (2019). Multipass Serpentine Cooling Designs for Negating Coriolis Force Effect on Heat Transfer: 45-deg Angled Rib Turbulated Channels. Journal of Turbomachinery 141(7).

Tanda, G. (2011). Effect of rib spacing on heat transfer and friction in a rectangular channel with 45 angled rib turbulators on one/two walls. International Journal of Heat and Mass Transfer 54(5-6), 1081-1090.

Viswanathan, A. K. and D. K. Tafti (2004, January). Detached eddy simulation of turbulent flow and heat transfer in a ribbed duct. In Heat Transfer Summer Conference 46911, 345-361. 